\title{
Short communication \\ Cyclodextrin nanosponges in the removal of organic matter to produce water for power generation
}

\author{
BB Mamba $^{1 *}$, RW Krause ${ }^{1}$, TJ Malefetse ${ }^{1}$, G Gericke ${ }^{2}$ and SP Sithole ${ }^{1}$ \\ ${ }^{1}$ Department of Chemical Technology, University of Johannesburg, PO Box 17011, Doornfontein, 2028, South Africa \\ ${ }^{2}$ ESKOM, ERID, Water Section, Private Bag 40175, Rosherville, Cleveland, South Africa
}

\begin{abstract}
The water treatment processes employed by coal-fired power stations do not completely remove most of the natural organics (volatile component) from the feed water used for power generation. Currently, polyaluminium chloride, polyelectrolyte and ion exchange resins are used to treat water at power stations. The effectiveness of water-insoluble cyclodextrin (CD) polymers in the removal of natural organics (volatile component), dissolved organic carbon (DOC) and total organic carbon (TOC) from water collected at a specific power plant is reported. Results obtained from this study show that, despite the usage of the treatment processes, natural organic species emanating from raw water still persist throughout the stages of the water treatment process. The polymers on the other hand demonstrated the ability to remove dissolved organic carbon (DOC) from raw water by as much as $84 \%$, whilst TOC removal was relatively low.
\end{abstract}

Keywords: organic pollutants, dissolved organic carbon (DOC), total organic carbon (TOC), cyclodextrin polymers, coagulants, ion-exchange resin

\section{Introduction}

The presence of organic pollutants in raw water is a major concern for a number of power plants and industries requiring ultra-pure water such as pharmaceutical and electronics sectors (Maughan et al., 2000). Power stations in South Africa generally acquire their water from neighbouring surface water sources and face similar problems in terms of the presence of organic pollutants. Also, this water often contains inorganic salts as well as natural organic matter (NOM). The concentration levels of organics depend very much on the catchment, soil properties and other industrial and agricultural activities in the area. Seasonal variations are also a key factor as they also contribute to the level of organics in water. As per Eskom's chemistry guidelines, water specifications illustrated in Table $\mathbf{1}$ have to be adhered to (Gericke, 2002).

On entering the boilers (Fig. 1), these organic impurities can be decomposed to short-chain organic acids and carbon dioxide through thermo-hydraulic processes $\left(540^{\circ} \mathrm{C}, 18 \mathrm{MPa}\right)$ (Aspden et al., 2002). The newly formed acids can cause a decrease in the $\mathrm{pH}$ of the boiler water depending on the level of contamination, which in turn can impact negatively on the steam quality.

To alleviate this problem Eskom, the electricity generating utility in South Africa, has developed a strategy which is aimed at producing water with reduced organic load. The approach in this strategy entails reducing organic loading in the water through the conventional treatment processes employed. These conventional treatment processes which have now been adopted by all power stations involve coagulation and flocculation of raw water followed by clarification, sand filtration and demineralisation

\footnotetext{
To whom all correspondence should be addressed.

+2711 559 6516; fax: +2711 5596425

e-mail:bmamba@uj.ac.za
}

\begin{tabular}{|l|c|}
\hline \multicolumn{3}{|c|}{ TABLE 1 } \\
Demineralisation water specifications for coal fired \\
power stations measured at the mixed bed outlet \\
(Gericke, 2002)
\end{tabular}

Key: ALARA: as low as reasonably achievable

(Aspden et al., 2000). These processes are illustrated in Fig. 1.

Herein, we report on the effectiveness of cyclodextrin polymers, synthesised in our laboratories (Mhlanga et al., 2007; $\mathrm{Li}$ and $\mathrm{Ma}, 2000$ ), in the removal of organic pollutants from water sampled at a specific power station. The effectiveness of these water-insoluble cyclodextrin polymers in the removal of organic species from water present in concentration levels as low as $\mathrm{ng} / \ell$ has been previously reported (Salipira et al., 2008; Mamba et al., 2007).

\section{Experimental}

\section{Sampling site}

Water samples $(20 \ell)$ were collected from each of the different sampling points at a specific power station (raw water outlet, clarifier outlet, potable water head tank inlet, cation exchanger outlet, weak base and strong base outlet) as shown in Fig. 1. It should be noted that only raw water was used in total organic carbon (TOC) and ultraviolet-visible (UV-Vis) analyses. 


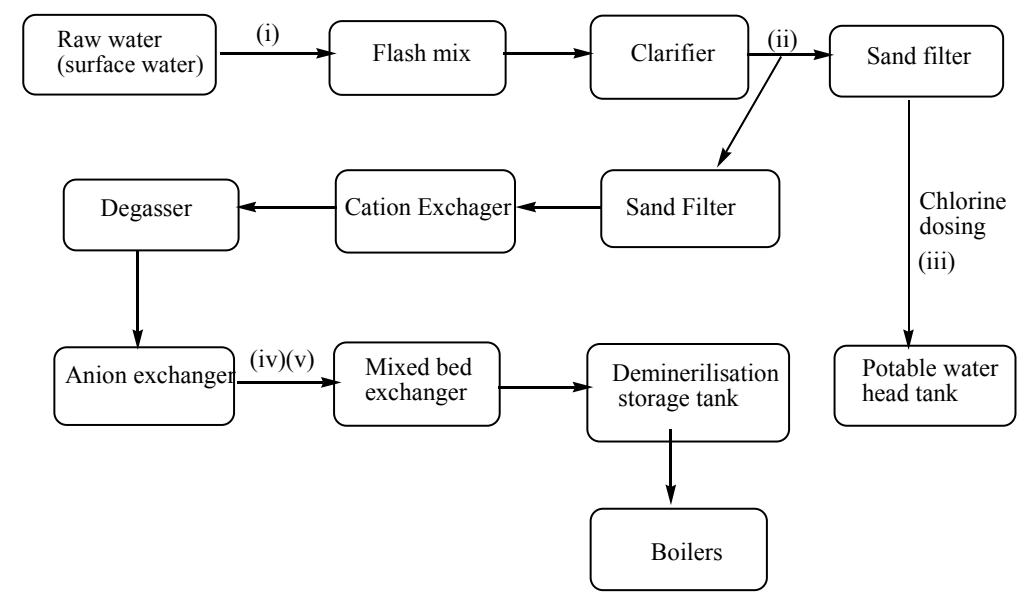

\section{Figure 1}

A representation of a power station water-treatment pathway showing the sampling points (i) raw water outlet, (ii) clarifier outlet, (iii) potable water inlet (iv) weak base outlet and (v) strong base outlet.

\section{Treatment of water samples with cyclodextrin polymers}

$\beta$-CD/TDI and $\beta$-CD/HMDI polymers (500 $\mathrm{mg}$ each) were loaded into an empty cartridge $\left(6 \mathrm{~cm}^{3}\right)$ and $500 \mathrm{~m} \ell$ of the water samples were passed through. Filtration was enhanced by the use of a vacuum pump or water aspirator. Water samples treated with cyclodextrin polymers were extracted using solid phase microextraction (SPME) and analysed using gas chromatography-mass spectrometry (GC-MS) as previously reported by Mamba et al (2007). UV-Vis and TOC analysis were also carried out before and after passing raw water through the polymers.

Polymers used in TOC and UV-Vis analyses were first washed with deionised water several times in order to reduce excess carbon from the polymer which would have caused an increase in the TOC values. The washed polymer was then used in the treatment of raw water samples.

\section{Ultraviolet-visible (UV-VIS) spectroscopy analysis of water samples}

A CARY-UV 50 was used for the analysis of raw water. The water sample was filtered through a $0.45 \mu \mathrm{m}$ polyethylene filter paper. The measurements were determined at a UV wavelength of $254 \mathrm{~nm}$. This was done in order to determine how much dissolved organic carbon (DOC) is present in the water samples. These analyses were carried out before and after passing raw water through the polymers.

\section{Total organic carbon (TOC) analysis of the water samples}

A Tekmar Dohrmann Apollo 9000 TOC combustion analyser was used for the analysis of the raw water sample. Aliquots of the water samples were poured into sample vials that have pierceable Teflon septum caps. The vials were then placed on the TOC analyser autosampler rack for analysis. Three replicates were run. Standard potassium phthalate samples of 1, 2, 5, 10 and $15 \mathrm{mg} / \ell$ were prepared and a calibration curve plotted. TOC concentrations were deduced from the calibration curve.

\section{Extraction technique}

A solid phase micro-extraction (SPME) device was used for the extraction of the analytes from the water samples collected from sampling points shown in Fig. 1. The extraction technique and procedure have been previously discussed by Mamba et al. (2007).

\section{Analysis of water samples using gas chromatography- mass spectrometry}

GC-MS analysis was carried out using a Varian CP-3800 capillary Gas Chromatograph coupled with a Varian Saturn 2000 Mass Spectrometer. This technique was used because of its ability to separate and uniquely identify organic compounds from a complex mixture. The one drawback of GC-MS is that only part of the organic load (i.e. volatile and semi-volatile organics) is analysed; nonetheless, it gives a good indication of the effectiveness of the cyclodextrin polymers

Water samples before and after treatment with cyclodextrin polymers were first extracted using SPME (Mamba et al., 2007). After extraction of organic compounds the SPME fibre was retracted from the sample and placed in the GC injector port to desorb for $3 \mathrm{~min}$.

\section{Results and discussion}

\section{Ultraviolet-visible (UV-VIS) and total organic carbon (TOC) analysis}

The UV-Vis analysis at $254 \mathrm{~nm}$ demonstrated the ability of the cyclodextrin polymers in the removal of dissolved organic carbon from the raw water sample (Table 2). $\beta$-CD/TDI polymer removed about $84 \%$ of DOC whilst $\beta-\mathrm{CD} / \mathrm{HMDI}$ removed about $73 \%$. These findings are comparable to those obtained from a water treatment plant when enhanced coagulation was applied; DOC removals of up to $90 \%$ were achieved (Gericke, 2002).

TABLE 2

$\%$ dissolved organic carbon removal from raw water

\begin{tabular}{|l|c|c|c|}
\hline Polymer & $\begin{array}{c}\text { Initial } \\
\text { absorbance }\end{array}$ & $\begin{array}{c}\text { Final } \\
\text { absorbance }\end{array}$ & $\begin{array}{c}\% \text { DOC } \\
\text { removal }\end{array}$ \\
\hline$\beta$-CD TDI & $0.268 \pm 0.001$ & $0.044 \pm 0.001$ & 84 \\
\hline$\beta$-CD HMDI & $0.268 \pm 0.001$ & $0.073 \pm 0.001$ & 73 \\
\hline
\end{tabular}

The TOC results shown in Table 3 demonstrate low levels of TOC removal from raw water which is far below the $60 \%$ removal when compared to enhanced coagulation.

\begin{tabular}{|l|c|c|c|}
\hline \multicolumn{4}{|c|}{ TABLE 3} \\
\hline Polymer & $\begin{array}{c}\text { Initial TOC } \\
\mathbf{m g} / \mathbf{l}\end{array}$ & $\begin{array}{c}\text { Final TOC } \\
\mathbf{m g} / \mathbf{l}\end{array}$ & $\begin{array}{c}\text { \% TOC } \\
\text { removal }\end{array}$ \\
\hline$\beta$-CD TDI & 3.01 & 2.84 & 5.6 \\
\hline$\beta$-CD HMDI & 3.01 & 2.48 & 17.6 \\
\hline
\end{tabular}


The high DOC and low TOC removals would mean that the polymers are effective in the removal of dissolved organic carbon rather than the particulate organic carbon which might be responsible for the lower percentage TOC removal. Therefore the cyclodextrin polymer technology can be positioned between the sand-filter and the cation exchanger. By doing so the clarifier will remove the solid particles whilst the polymer removes DOC that cannot be effectively removed by the clarifier.

\section{Quantitative analysis of the water samples using SPME and GC-MS}

The presence of volatile organic compounds in raw water before treatment with cyclodextrin polymers was confirmed by the appearance of signals in the GC-MS chromatogram illustrated in Fig. 2A. The chromatogram showed reduced intensities after the coagulation and flocculation process (Fig. 2B). Approximately $50 \%$ removal was obtained when taking into account the reduction in ion counts from raw water to the clarifier outlet. However, the persistence of these signals from water sampled at the clarifier outlet (Fig. 2B) even after treatment with polyaluminium chloride and the polyelectrolyte is evidence that the coagulation and the flocculation processes do not completely remove these pollutants from the water. GC-MS chromatograms of the other three sampling points (potable water inlet, strong-base and weak-base outlets) showed a similar trend. This indicates a persistent presence of volatile organics in both potable and demineralised water. Additionally, this serves to demonstrate that the water treatment methods used in the removal of organic impurities need to be improved. The cyclodextrin polymers can be used in conjunction with Eskom's existing technology.

It can be noted that after passing the raw water through $\beta$-CD/TDI polymer, an almost complete removal of the volatile organics was observed as shown in Fig. 2C. A similar trend was noted when the raw water was passed through $\beta-C D / H M D I$ polymer. A removal efficiency of $90 \%$ (volatile and semi-volatile organics) was estimated based on the ion counts before (Fig. 2A) and after treatment (Fig. 2C) with the cyclodextrin polymers when taking into account the GC-MS analysis.

\section{Qualitative analysis of the water samples using SPME and GC-MS}

Three volatile organics were inferred using the MS online library (NIST library). The compounds are 4-chlorophenylphenylether, 5-hydroxyindole and ethoxyquin. While 5-hydroxyindole and ethoxyquin were detected in all sampling points the presence of 4-chlorophenyl-phenylether was only observed in the last two sampling points (weak base and strong base outlet). 4-Chlorophenyl-phenylether is generally used as a dielectric fluid and can be released in the environment through its use in capacitors. Ethoxyquin is utilised as a fungicide, preservative in animal feed and as an anti-degradation agent. 5-Hydroxyindole is found in dye-making industries, agrochemicals and perfumes. All these compounds are probably from other industrial activities, including agriculture, which are eventually released into the river systems through runoff and soil leaching.

\section{Conclusion}

The results that emanated from the DOC and TOC analysis of the raw water after the use of the cyclodextrin polymers indicated that the cyclodextrin polymers achieve a similar reduction in DOC as the currently used enhanced coagulation. We

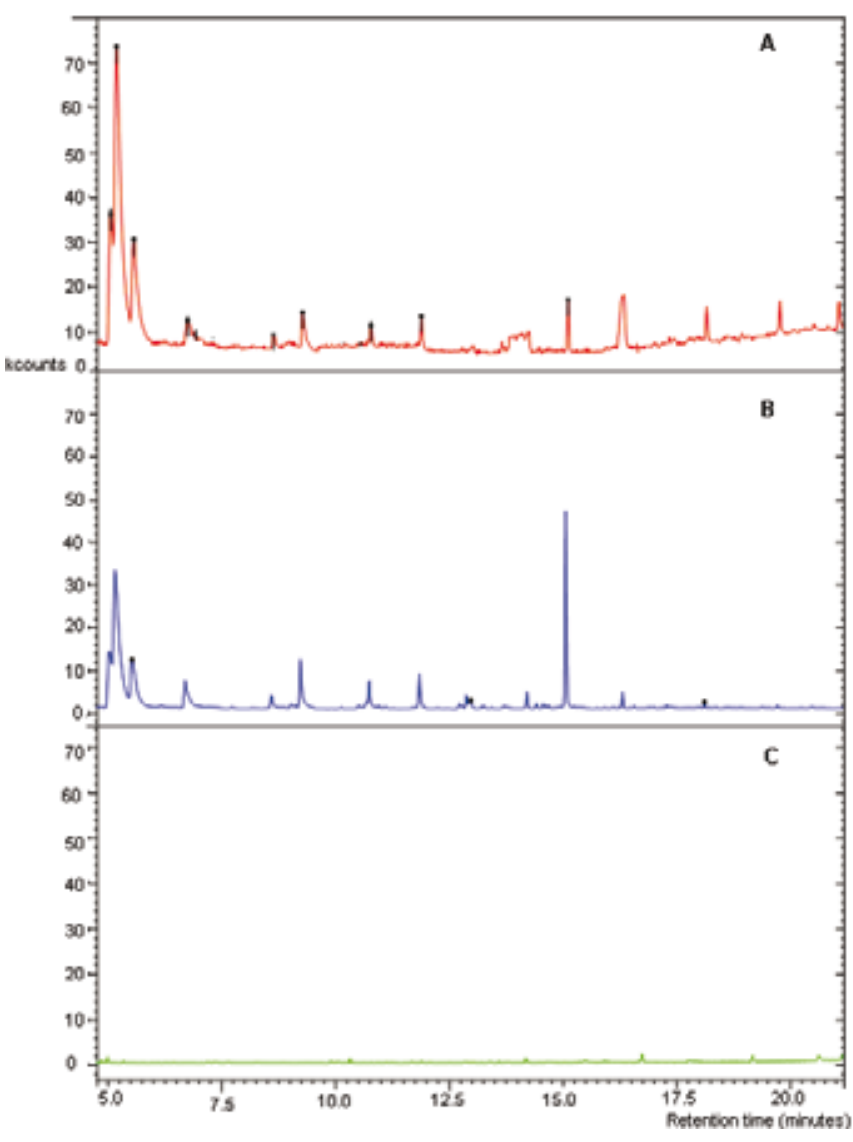

Figure 2

GC-MS chromatograms of A: raw water before treatment with cyclodextrin polymers and polyaluminium chloride with minimal polyelectrolyte. B: Clarifier after treatment with polyaluminium chloride and minimal polyelectrolyte C: Raw water outlet after passing through $\beta-C D / T D$ I polymer

therefore propose that the polymers can be utilised at site points between the sand-filter and cation exchanger (refer Fig. 1). The clarifier would remove the bulk of the particulate and dissolved organic carbon whilst the rest could be removed by the cyclodextrin polymer before the water enters the demineralisation plant. While Eskom is continually evaluating new technologies, enhanced coagulation is the one that is in current practice. It is evident that cyclodextrin polymers achieve a similar DOC removal as enhanced coagulation but it is difficult to make a comparison of both technologies due to unknown costs of scaling up of the cyclodextrin technology. On the other hand no estimation of costs for enhanced coagulation is available either.

\section{Acknowledgements}

Funding for this research from the University of Johannesburg, National Research Foundation (NRF), the Water Research Commission (WRC) and Eskom Tertiary Education Support Programme (TESP) is gratefully acknowledged.

\section{References}

ASPDEN JD, DENDEKIND I, GERICKE G and WILSHIRE S (2002) The impact of natural organic matter and chemical selection on the water treatment plant at Kendal power station. Int. J. Electr. Heat Gener. 82 82-85. 
GERICKE G (2002) Plant optimization: A key factor in minimising natural organic matter in power station make-up water. $V G B$ PowerTech. 3 93-96.

LI D and MA M (2000) Nanosponges for water purification. Clean Products Proc. 2 112-116.

MAMBA BB, KRAUSE RW, MALEFETSE TJ, MHLANGA SD, SITHOLE SP, SALIPIRA KL and NXUMALO EN (2007) Removal of geosmin and 2-methylisorboneol (2-MIB) in water from Zuikerbosch Water Treatment Plant (Rand Water) using $\beta$-cyclodextrin polyurethanes. Water SA 33 223-228. http://www.wrc.org.za/downloads/watersa/2007/Apr\%2007/2002.pdf

MAMBA BB, KRAUSE RW, MALEFETSE TJ and NXUMALO EN (2007) Monofuctionalized cyclodextrin polymers for the removal of organic pollutants from water. Environ. Chem. Lett. 5 79-84.
MAUGHAN EV, GERICKE G and ROSCOE G (2000) The measurement of TOC in the cycle fluid modern power plants and correct selection of monitoring equipment. Power Plant Chem. 2 219-222.

MHLANGA SD, MAMBA BB, KRAUSE RW and MALEFETSE TJ (2007) Removal of organic contaminants from water using nanosponge cyclodextrin polymers. J. Chem. Technol. Biotechnol. 82 382-388.

SALIPIRA KL, MAMBA BB, KRAUSE RW, MALEFETSE TJ and DURBACH SH (2008) Cyclodextrin polyurethanes polymerised with carbon nanotubes for the removal of organic pollutants in water. Water SA 34 113-118. http://www.wrc.org.za/downloads watersa/2008/2198.pdf 